九州大学学術情報リポジトリ

Kyushu University Institutional Repository

Characterization and Analysis of Mechanical Properties of Short Carbon Fiber Reinforced Polyamide66 Composites

Choudhari, Dilip

Department of Mechanical Engineering

Kakhandki, Vyasraj

Department of Mechanical Engineering

https://doi.org/10.5109/4742120

出版情報 : Evergreen. 8 (4)，pp.768-776，2021-12. 九州大学グリーンテクノロジー研究教育センター バージョン：

権利関係 : 


\title{
Characterization and Analysis of Mechanical Properties of Short Carbon Fiber Reinforced Polyamide66 Composites
}

\author{
Mr. Dilip Choudhari ${ }^{1, *}$ \\ Dr. Vyasraj Kakhandki ${ }^{2}$ \\ 1,2 Department of Mechanical Engineering \\ ${ }^{1,2}$ Trinity College of Engineering \& Research, Pune, India \\ *Email-dilipchoudhari2009@gmail.com
}

(Received March 29, 2021; Revised September 9, 2021; accepted November 11, 2021).

\begin{abstract}
In recent years, Short Carbon Fiber (SCF) reinforced Polyamide66 (PA66) composites materials have increased their demand in automotive and aircraft industries due to their superior mechanical properties. Firstly, different specimens were prepared of 00, 10, 20, 30, 40, 50, 60\% weight of SCF content in PA66. As per ASTM standards, tensile, flexural, compression, and density specimens were manufactured on an injection molding machine. Then, tensile, flexural, compression specimens are tested on UTM and density specimen tests carried out directly on mass and volume relation. Secondly, on advanced micrographs Field Emission Scanning Electron Microscopy (FESEM), tensile, flexural, and compression specimens were tested. In the tensile test, it was found that most SCFs were pulled out and some agglomerated from the matrix instead of breaking. The flexural test showed that the debonding of SCF from the grid is because of the matrix uncovered close to the fiber surface. Cleavage was observed due to a plastic twist of the matrix after SCF peeling. In the compression test, extreme adhesion and a compatible bond between SCF and matrix are observed. It is pushed together with higher compressive force, only small grooves and cavities are observed and not much damage. Further, observed that all specimens have a strong interface, adhesive bond, no differences in matrix holes sizes, even fibers distribution, and no segregation.
\end{abstract}

Keywords: Short Carbon Fiber, Polyamide66, Injection Molding, Mechanical Properties, FESEM.

\section{0. Introduction}

With the advancement of engineering materials technology, advanced composites materials are developed for applications in diversified fields of space, automobiles, industries, etc. SCF reinforced PA66 composites exhibit outstanding tailor-made properties of mechanical like good tensile strength, low density, modulus of elasticity, and better wear resistance. Analysis and study of their characteristics and potential as alternative materials to replace metallic materials are carried out. SCF can be easily mixed, interfacial, and compacted with PA66. It can be used in aerospace, transportation, automotive, industrial application, sports goods, marine, and defense industries. Also has potential for specific applications in pharmaceutical machinery, food processing machinery, printers, and Xerox machines.

Fiber-reinforced PA66 composites are light in weight, wear-resistant, and used to manufacture gears, bearings due to improved mechanical properties, compared to PA66 ${ }^{1)}$. SCF-reinforced thermoplastics are used for the structural parts of automobiles and aircraft due to their good mechanical properties, such as high rigidity, strength, recyclability, and short molding time ${ }^{2), 3}$. Adhesive bonds have a strong influence on the mechanical properties of particulate metal foams ${ }^{4)}$. The study investigates the fracture behavior and mechanical properties of a polyamide6 unidirectional epoxy resin reinforced with SCF ${ }^{5)}$. Shape memory polymers improve shape recovery stress and mechanical properties to match switch temperature and biomedical properties ${ }^{6), 7)}$. Polyamide6 composite's thermal and mechanical properties are less superior than the SCF and glass fiber reinforced polyamide 6/clay nanocomposites 8). Unidirectional SCF and epoxy reinforced polyamide is fabricated using different matrix systems and same SCF ${ }^{9}$. Mechanical and static fatigue properties of $20 \%$ by weight by SCF reinforced polyamide6 are investigated on both unnotched and notched specimens ${ }^{10)}$. The manufacture of composite in various combinations with tailored strength properties, high fatigue, toughness, oxidation resistance capabilities, high temperature, and wear provides an excellent choice in engineering applications 11). SCF reinforced the melt blending process manufactures polyamide 6 composites, and their influence of content and 
fiber length on the mechanical and thermal properties is investigated ${ }^{12)}$. During the time process hot compression molding, fiberglass Polypropylene composites with various stacking conditions and volume portions are utilized ${ }^{13)}$. Among all the manufacturing methods of composite, injection molding has improved processability and higher efficiency ${ }^{14}$. Long SCF, the injection molding machine produces reinforced polyamide 6 composites, a novel kind of core pellets by a single screw extruder ${ }^{15)}$. The outcome shows that as the SCF content enhances in polyamide, the composite increase's fracture strength and tensile properties increase ${ }^{16)}$. Extrusion and pultrusion techniques are employed in the continuous melt compounding and discontinuous SCF reinforced PA66 composite ${ }^{17)}$. It developed SCF/PA12 composite powder for high production by selective laser sintering to improve its performance ${ }^{18), 19)}$.

SCF built up polymer composites are additionally utilized in hardware, aeronautic design, tidal turbine blades, corrosion resistant and biomedical 20), 21). Hybrid fiber-reinforced polymer (HFRP) composites have received tremendous attention due to their higher strength and lighter weight ${ }^{22)}$. 30, 30, and 60 percentage chopped short carbon fiber reinforced nylon 66 for tensile, flexural, and compression strength respectively is the optimum by the rule of mixture method ${ }^{23)}$. PA and SCF composites exhibit high tensile strength, interfacial shear strength, fatigue resistance, wear resistance, corrosion resistance, elastic modulus, thermal conductivity, electrical conductivity, chemical inertness, and thermal stability as compared to untreated SCF 24), 25). Thermoplastic composites are easier for processability and recycling convenience than thermosetting composites ${ }^{26)}$. Expanding the SCF weight part in Acrylonitrile Butadiene Styrene (ABS) from 10 to $30 \%$ weight brings about worked on tensile strength and tensile modulus ${ }^{27)}$. Temperature is one of the most significant parameters influencing the mechanical properties, the contact state of the boundary, and as result friction, fatigue, and wear behavior of polymers 28), 29). Thermal expansion in the transverse and flow direction decreases as filler content increases for all composites ${ }^{30}$.

There is also a need for polymer composites with natural fillers as renewable sources and biodegradable materials towards environmental, pollution issues, recycled mixed plastics ${ }^{31), 32)}$. Welded joints of short fiber supported polymer composites, exhaustion examination and life assessment strategies are utilized 33). Polyamide resin is a beneficial SCF reinforced thermoplastics matrix because of less cost and good bonding properties ${ }^{34)}$. Conventional materials are recently replaced by reinforcing nanoscale for their smart, multi-functionality, superior properties, and novel ${ }^{35)}$. Polymer and carbon-based materials are used in air conditioning applications as water absorption ${ }^{36)}$. Corrupting the hardness and modulus by doping of Si particles as contrasted and undoped films ${ }^{37)}$. SEM observations of fracture surfaces reveal SCF's fine spread, the strong interfacial adhesion between matrix and fibers, and shear failure ${ }^{38), 39)}$. Dry composites show better tensile strength, better modulus of elasticity, better elongation, better density for weight reduction, and better stability under SEM ${ }^{40)}$. In SEM, voids influence the mechanical properties because of the presence of voids or holes in composites, and carbon materials can be improved by changing microdomain and nanoscale space structures ${ }^{41), 42)}$. SEM observation shows that the changes with maleated elastomers improved the interfacial adhesion between polyamide6 and SCF ${ }^{43)}$. The results show that PA66 composites containing glass fiber transition and short glass fiber from ductile to brittle behavior as a function of hydrolysis time in resin ${ }^{44}$.

The literature survey observed that SCF-reinforced thermoplastics could be used for structural, composition, and automobiles to improve mechanical properties and biomedical applications. Adhesive bonds have a powerful effect on the mechanical properties of composites. Composites are tailor-made with desired strength properties. Fatigue, toughness, temperature, wear, and oxidation resistance are excellent choices in engineering applications. Tensile, flexural, and compression strength of composite materials are increased as compared to untreated SCF. There is a need to develop polymer composites with natural fillers as renewable sources and biodegradable materials due to environmental, pollution issues, recycled mixed plastics. There is a trend to replace conventional materials with reinforced composite materials due to their smart, novel, superior properties, and multi-functionality.

The novelty feature of this research is the original development of new materials. The composite testing specimens using SCF and PA66 material are investigated. SCF is used wide range from $00 \%$ to $60 \%$ in PA66 to determine the tailor-made optimum mechanical properties because of replacing the convectional metals for engineering applications. FESEM test, systematically find out failure analysis for tensile, flexural, and compression specimens. Also measured the average diameter, length of SCF, and average hole diameter in a matrix. It can use for high magnification factors so that more clear image micrographs know specimen failure analysis. It shows that specimens have a strong compatible interface, adhesion bonding, fibers distribution is uniform, and no segregation is observed.

\subsection{Material Characteristics and manufacturing methods}

\subsection{Short Carbon Fiber \& Polyamide66}

SCF is light in weight twice stiff and about 5.5 times stronger than steel. It is 6 microns in diameter and $6 \mathrm{~mm}$ in length supplied by Formosa Plastics, Taiwan, as shown in Fig.1(a). Pellet PA66 has been recognized as an excellent thermoplastic composite that is compatible with 
short carbon fiber. Due to its low coefficient of friction, it has excellent wear resistance and is selflubricating. It is typically used in bearings, gears, handles, cams, bushings, sheaths, cables, and wires. Solvay Chemical South Korea supplies have pellets form with an average length of $2.00 \mathrm{~mm}$ and a diameter of $1.50 \mathrm{~mm}$, as shown in Fig.1(b).

The reinforcement of SCF increase by weight fraction in PA66 composites results in enhanced tensile, compressive, flexural strength. SCF length does not affect the hardness, tensile strength, and modulus but increasing SCF length increases the strain at the composites breakpoint. The fracture strain of long fiber composite is less than the short fiber composites, but the modulus of elasticity and tensile strength is high.

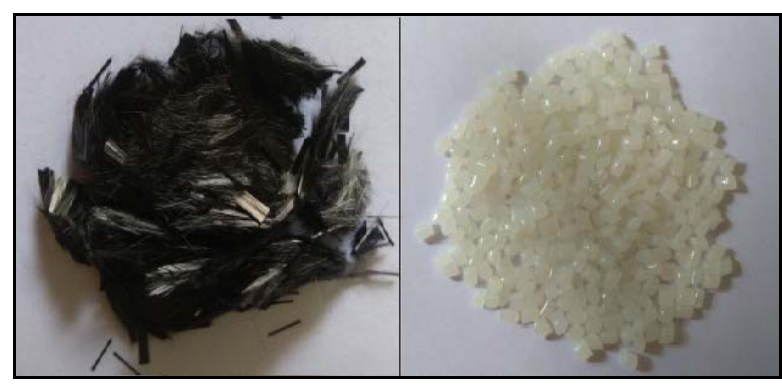

(a) Short carbon fiber

(b) Polyamide66

Fig.1: Materials (a) Short carbon fiber, (b) Polyamide66.

\subsection{Manufacturing Methods}

As per ASTM D 638-14, ISO 178, ASTM D-695, and ASTM D-792 standards tensile, flexural, compression, and density tests, respectively specimens are manufactured on the injection molding machine. SCF and PA66 materials are preliminarily dehumidified in the oven at $80^{\circ} \mathrm{C}$ for 4 hours and mixed in the ratios.

The mixture of material passed through a single extruder works at a speed of $100 \mathrm{rpm}$ for 3 minutes and then the mixture slowly moves forward with a screw plunger. It is pushed into a barrel heating chamber where it melts. Then, the molten plastic is forced through a nozzle at the temperature range of $285-320^{\circ} \mathrm{C}$. The rests against the mold so that it can enter the mold cavity as a sprue and gate system. The mold filled with the molten mixture is cooled for 6 seconds to solidify the specimen, and the manufacturing procedure of specimens is shown in Fig. 2.

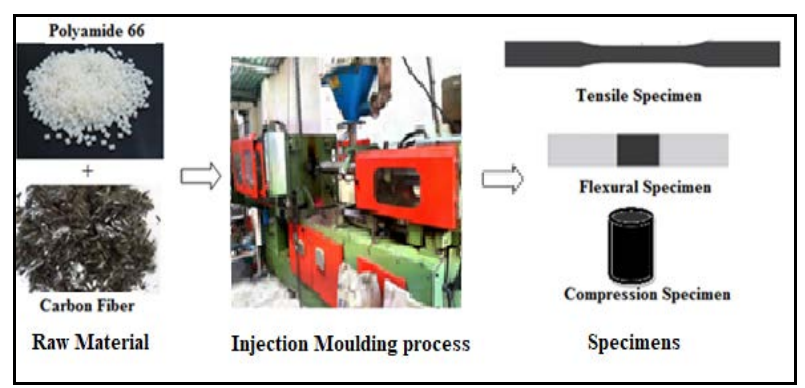

Fig. 2: The manufacturing procedure for specimens.

\subsection{Experimental Approach}

SCF reinforced PA66 composite tensile, flexural, compression, and an injection molding process manufactures density specimens. Different specimens are prepared by adding 0,10,20,30,40,50,60 \% weight of SCF content and remaining PA66 in the mixture. The SCF orientation and distribution are random and discontinuous. These tests are conducted in a laboratory controlling the environment conditions at temperature $23^{\circ} \mathrm{C}$, humidity $55 \%$ for all tests.

\subsection{Tensile strength test}

The tensile strength test is performed as per the standard of ASTM D 638-14 on UTM, Tinius Olsen, UK. These specimens are tested for static tensile fatigue tests. The dimensions are straight-sided gauge length, $L_{G}=100 \mathrm{~mm}$, width, $\mathrm{w}=13 \mathrm{~mm}$, and thickness, $\mathrm{t}=2.12 \mathrm{~mm}$. These tests are conducted on "dumbbell" or "dogbone" shaped test specimens on the UTM machine using a test cross speed of $50 \mathrm{~mm} / \mathrm{min}$ as determined by the material specifications. Specimens are subjected to a controlled tension until they fail. The average results for each composite are calculated.

\subsection{Flexural strength test}

The flexural strength tests are performed as per ISO 178 standard on the UTM. The specimens' dimensions are gauge length, $\mathrm{L}_{\mathrm{G}}=125.89 \mathrm{~mm}$, width, $\mathrm{w}=13.84 \mathrm{~mm}$, and thickness, $\mathrm{t}=2.12 \mathrm{~mm}$. This test is conducted on rectangular-shaped test specimens to investigate the specimens' flexural behavior at a constant cross speed of $2.00 \mathrm{~mm} / \mathrm{min}$. This test is performed on three-point, and the specimen is deflected in the center of the span at a constant rate until a break occurs. On the specimen's outer surface or until a maximum elongation reaches $5 \%$, whichever occurs first.

\subsection{Compression strength test}

The compression strength tests are performed as per ASTM D 695 standards on the UTM machine. The specimens for static-compression and compressioncompression fatigue tests are used to eliminate specimen buckling risk due to the applied compressive load. The detailed dimensions of the specimen are length, $L=30.70$ $\mathrm{mm}$, and diameter, $\mathrm{d}=10 \mathrm{~mm}$. The specimen is placed compression with its parallel faces in contact between compression plate plates and then compressed at a uniform speed of $1.300 \mathrm{~mm} / \mathrm{min}$. An extensometer is used to measure specimen parameters. The maximum load is recorded along with gradient stress-strain data. When the compressive load is relatively low and uniform, it is subjected to material behavior, and compressive strength is generated.

\subsection{FESEM test}

The advanced microstructure FESEM test has been carried out for the tensile, flexural, and compression specimens. In this test, the failure of specimens at their 
cross-section area has been investigated. Different 00, 10, 20, 30, 40\% weight fractions of SCF in PA66 of specimens are analyzed. In FESEM, specimen scale of $20 \mu \mathrm{m}$ and magnification factor 1000, 2500, 5000 has been used.

\subsection{Density test}

As per the ASTM-D-792 standard density test of specimens has been performed. The specimens are prepared into dimensions of $25.40 \mathrm{~mm} \mathrm{X} 25.40 \mathrm{~mm} \mathrm{X}$ $2.12 \mathrm{~mm}$. The density of the specimen is directly related to the mass and volume and is defined by the following equation:

Density $=$ mass/volume

An electronic balance machine measures the mass of the specimen. When the specimen is immersed in water is displaced, the volume of displaced water is measured, equal to the specimen's volume. The specimen for the density test is shown in Fig.3.

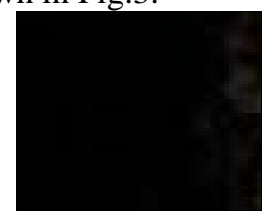

Fig.3: Specimen for density test

\subsection{Results and discussion}

Mechanical properties of SCF/PA66 composite for different $00,10,20,30,40,50,60 \%$ weight fraction of SCF content in PA66 composite are analyzed. The mechanical properties of tensile, flexural, compression, the density of specimens are analyzed. The composite material failure has been investigated by advanced microstructure FESEM test for the tensile, flexural, and compression specimens.

\subsection{Tensile strength}

As per ASTM-D-638-14, a standard tensile specimens test has been performed on UTM. A tensile strength indicates the ability of a material to withstand a greater tensile force. When the specimens are manufactured and tested the percentage of SSCF content increases from $00 \%$ to $40 \%$ by weight, tensile strength increases from 42.34 to $105.54 \mathrm{MPa}$. Further, with SCF increases up to $60 \%$, tensile strength considerably drops down to $81.53 \mathrm{MPa}$. Tensile specimens break near mid position. The specimens before and after failures are as shown in Fig.4. Specimens tensile test results by physical testing as shown in Fig. 5.

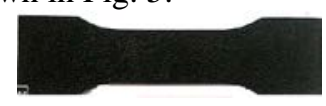

(a)Before failure

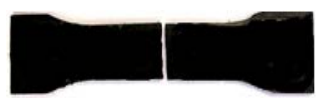

(b) After failure
Fig.4: Tensile Strength Test Specimen: a) Before failure, b) After failure.

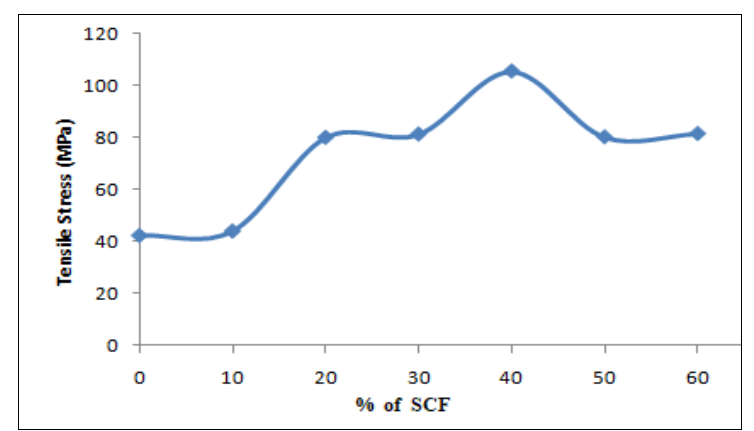

Fig.5: Tensile stress vs. PA66 and \% of SSCF in PA66.

\subsubsection{FESEM tensile analysis}

After the tensile tests, FESEM micrographs were taken of the fractured surfaces of the 10, 20, 30,40\% SCF/PA66. FESEM images using magnification 1000, 2500, 5000, and specimen scale of $20 \mu \mathrm{m}$ are shown in Fig.6. There is poor interfacial bond adhesion between SCF and PA66 with lower fiber content. It was observed that when carbon content increases from $10 \%$ to $40 \%$, more short carbon fibers are distributed in a matrix, and strength increases. If the SCF content increases beyond $40 \%$, it can be recognized as the fiber and matrix's interfacial adhesion. It is seen that most fibers get pulled out and some agglomerated from the matrix instead of breaking. It was found that the average diameter of holes in the matrix $(7.339 \mu \mathrm{m})$ is more than the average fiber diameter $(6.065 \mu \mathrm{m})$ when the fibers attempt to withdraw. It is also seen that fibers distribution throughout the matrix is uniform.

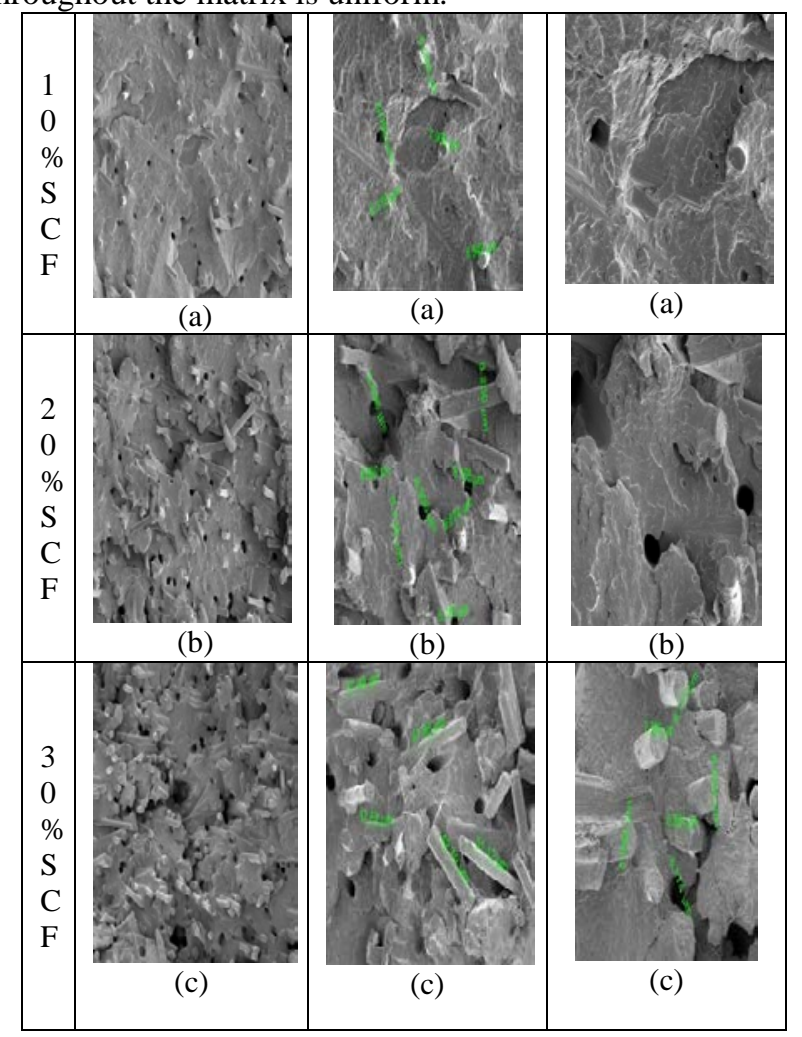




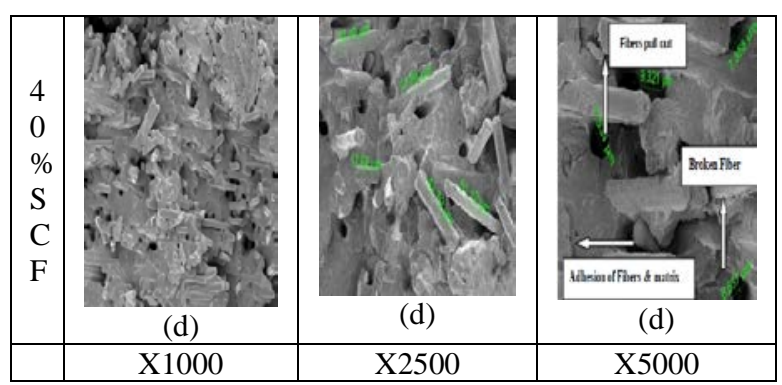

Fig.6. FESEM micrographs of failure surface specimens of SCF/PA66 composite after the tensile test:

a) SCF/PA66:10\% SCF (X1000, 2500, 5000),

b) SCF/PA66:20\% SCF(X1000, 2500, 5000),

c) SCF/PA66:30\% SCF(X1000,2500, 5000),

d) SCF/PA66:40\%SCF(X1000, 2500, 5000).

\subsection{Flexural strength}

Flexural specimens test as per ISO 178 standard is performed on UTM. A high value for the flexural strength indicates that the specimens have a higher flexural strength, and the fiber can withstand the maximum part of the loads. As specimens were made and tested, the percentage of SCF content increases from 00 to $40 \%$ by weight, and the flexural strength increases from 9.14 to $84.80 \mathrm{MPa}$. Further, with increases of SCF up to $60 \%$, tensile strength considerably drops down to $81.10 \mathrm{MPa}$. The flexural specimens before and after failure are as shown in Fig.7. Specimen flexural test results by physical testing as shown in Fig. 8.

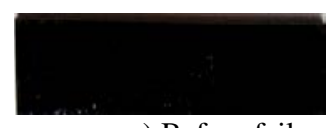

a) Before failure

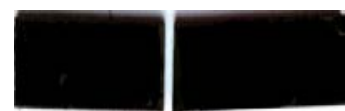

b) After failure
Fig.7: Flexural Strength Test Specimen: a) Before failure, b) After failure.

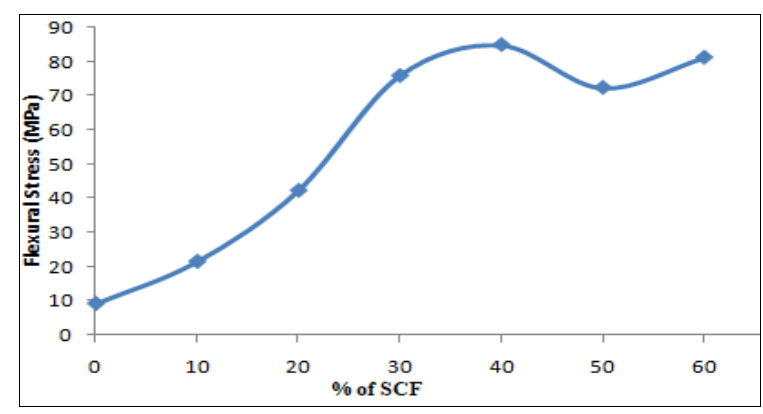

Fig.8: Flexural stress vs. PA66 and \% of SSCF in PA66.

\subsubsection{FESEM flexural analysis}

After the flexural tests, FESEM micrographs were taken of the fractured cross-section surfaces of the 10 , 20, 30, 40\% SCF/PA66. FESEM images at a magnification of 1000, 2500, 5000, and $20 \mu \mathrm{m}$ scales of specimens are shown in Fig.9. The SCF debonding from the PA66 matrix is due to matrix material exposed near the fiber surface while SCF is removed from the matrix. Cleavage was observed due to plastic deformation of the PA66 matrix after the fiber debonding. It is seen that the volume fraction of SCF reinforcement increases with increasing weight percentage, and due to the high weight fraction of fiber, the fiber starts coagulating. There is no effect on the size and shape of fibers, no differences in matrix hole sizes after the flexural test. SCF is evenly distributed, and no segregation is observed. More severe damage to the fiber, crushing, fragmented fibers, and delamination between SCF and PA66 were observed. Dark circles appeared between fibers and matrix, probably due to the matrix's local deformation around the fibers. Some fibers are agglomerated, and the bond strength between matrix and SCF weakens.

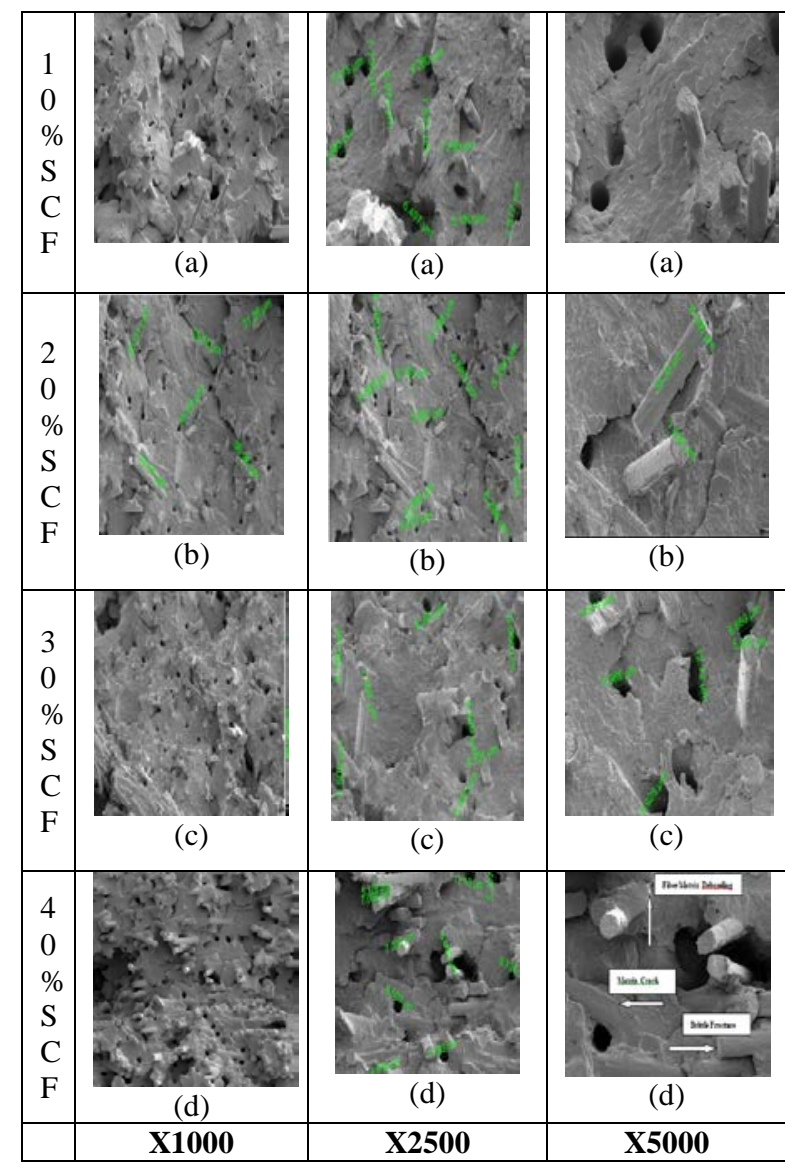

Fig.9: FESEM micrographs of failure surface specimens of SCF/PA66 composite after the flexural test: a) SCF/PA66:10\% SCF (X1000, 2500, 5000),

b) SCF/PA66:20\%SCF(X1000,2500,5000),

c) SCF/PA66:30\% SCF(X1000,2500, 5000),

d) SCF/PA66: 40\% SCF(X1000, 2500, 5000).

\subsection{Compression strength}

Compression specimens test as per ASTM D 695 standard has been performed on UTM. A high compressive strength value indicates that the specimens have high compressive strength, and the fiber can withstand the maximum part of the load. As the percentage of SCF content increases from $00 \%$ to $60 \%$ by weight, the flexural strength increases continuously from 91.07 to $124.97 \mathrm{MPa}$ by specimen tests. The compression strength specimens 
before and after failure are as shown in Fig.10. Compression test results by specimens' physical testing as shown in fig. 11.

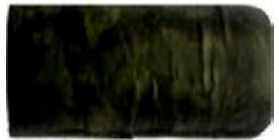

a) Before failure

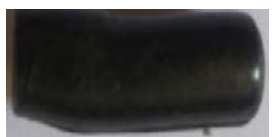

b) After failure
Fig. 10: Compression Strength Specimen: a) Before failure, b) After failure.

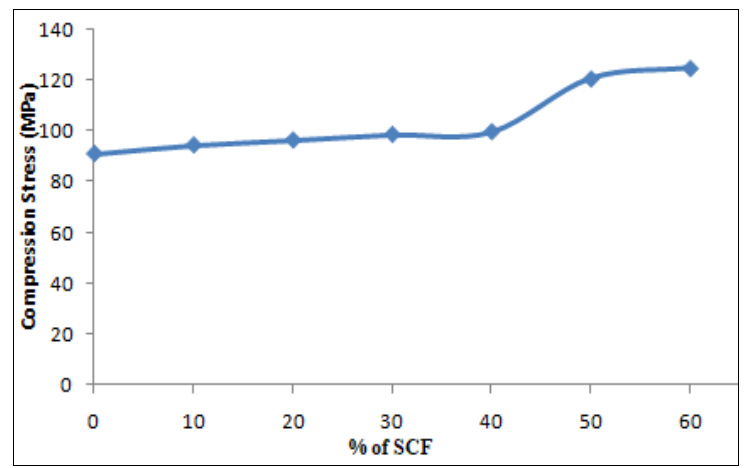

Fig.11: Compression stress vs. PA66 and \% of SSCF in PA66.

\subsubsection{FESEM compression analysis}

After the compression tests, FESEM micrographs were taken of the longitudinal fractured surfaces of the 10, 20, 30, 40, 50\% SCF/PA66. FESEM images at magnification 1000, 2500, 5000, and $20 \mu \mathrm{m}$ scales of specimens are shown in Fig.12. It is seen that by increasing the contents of SCF, and interparticle distance of fibers decreases and begins coagulating. There is a strong interface, and compatibility between matrix and fiber occurred. The SCF is evenly distributed in the matrix, and no segregation is observed. In this test, a strong adhesive bond between the matrix and SCF is observed. During the compression test, PA66 and SCF are pushed together with higher compressive force, less damage, and only small grooves and voids observed.

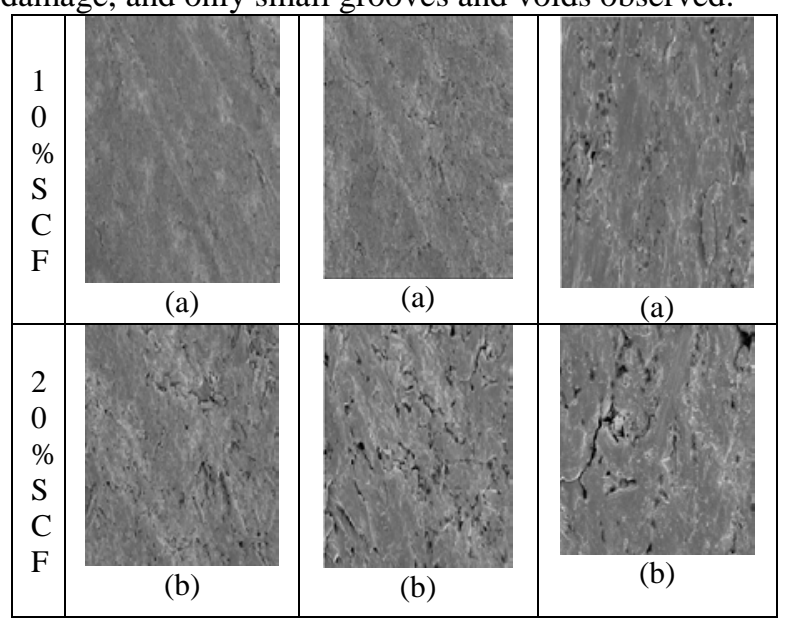

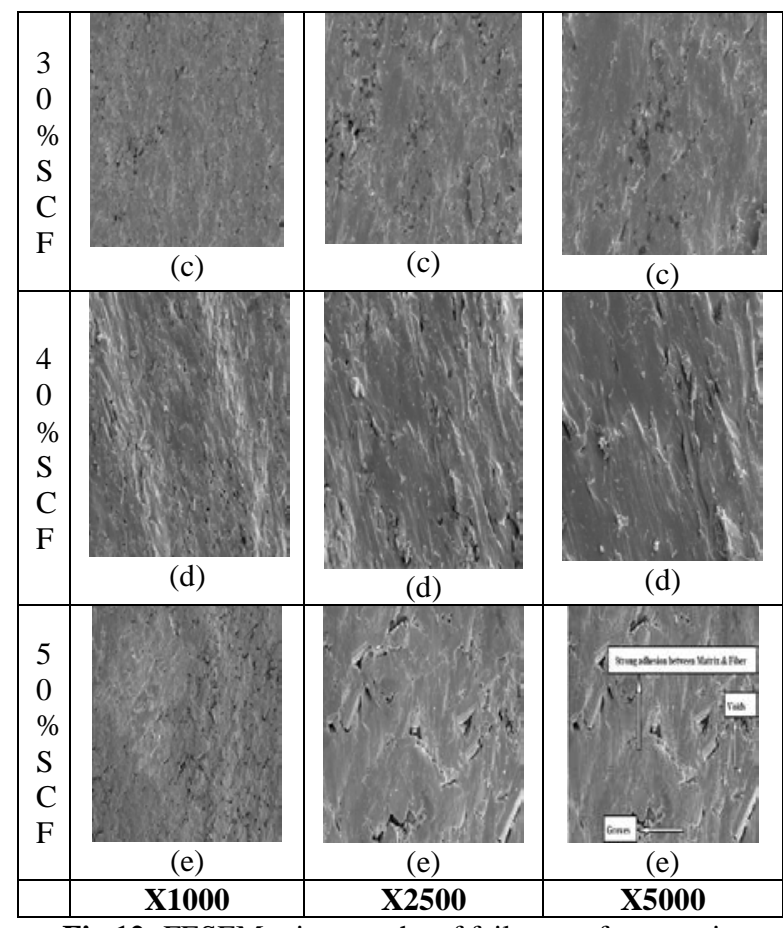

Fig.12: FESEM micrographs of failure surface specimens of SCF/PA66 composite after compression test: a)SCF/PA66:10\% SCF (X1000, 2500, 5000), b) SCF/PA66:20\% SCF(X1000, 2500, 5000), c) SCF/PA66:30\% SCF(X1000, 2500, 5000),

d) SCF/PA66:40\%SCF(X1000, 2500, 5000). e) SCF/PA66:50\%SCF(X1000, 2500, 5000).

\subsection{Density}

A density test of specimens as per the ASTM-D-792 standard has been performed. When testing specimens, the density increases continuously from 1032 to $1412 \mathrm{Kg} / \mathrm{m} 3$ as the percentage of SCF increases 00 to 60 weight\% as shown in Fig.13. The density of composite specimens was to be 5.54 times less than carbon steel.

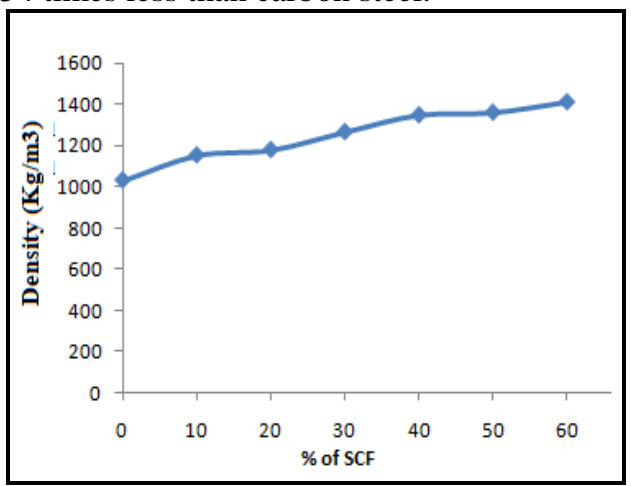

Fig.13: Density Vs. PA66 \& SSCF/PA66 composite.

\subsection{Conclusion}

Mechanical properties of composites test specimens by varying \% weight fraction of SCF in PA66 in a wide range from $0 \%$ to $60 \%$ are estimated. It was investigated for tensile and flexural strength; the composites specimens with $40 \%$ SCF exhibited the best retention of mechanical properties. Further, up to $60 \%$ weight fraction of SCF in PA66, the strength increases continuously or remains 
almost constant.

FESEM, composite specimens of tensile, flexural, and compression were tested on advanced micrographs. Most fibers were pulled out in the tensile test and some agglomerated from the PA 66 matrix instead of fractures. The average fiber diameter $(6.065 \mu \mathrm{m})$ is less than the average diameters of holes in the matrix $(7.339 \mu \mathrm{m})$ after fibers are pulled out. SCF debonding from the matrix is due to matrix material exposed near the flexural test's fiber surface. A brittle failure was observed due to plastic deformation of the matrix PA66 after the fiber debonding. Severe damage to SCF, fragmented fibers, crushing, and delamination between SCF and PA66 were observed. Dark circles appeared between matrix and fibers, probably due to local deformation of the matrix around the fibers.

A strong adhesive bond between the SCF and matrix was observed during a compression test. PA66 and SCF are pushed together with higher compressive force, little damage, and only small observed thickets and voids. It was also observed PA66 is tough in compression, but fragile in tension, whereas SCF is tough in tension, but fragile in compression; however, a combination of the two makes the bond stronger in both. It was estimated in the density test, the weight of composite specimens was 5.54 times less than carbon steel.

\section{Acknowledgments}

The authors thank S.S.Polymers for assisting manufacture and specimens testing at Auto Cluster and FESEM test at CIF, SPPU, India.

\section{References}

1) Santi Swarup Mohanty, Akshaya Kumar Rout, Dipak Kumar Jesthi, Bharat Chandra Routara, Ramesh Kumar Nayak, "Evaluation of mechanical and wear performance of glass/short carbon fiber reinforced polymer hybrid composite,” Materials Today: Proceedings, 5 (9)19854-19861(2018). doi.org/10.1016/j.matpr.2018.06.350.

2) Xiaosong Huang, "Review-Fabrication, and Properties of Short carbon fibers,” MDPI Journal Material, 2 (4) 2369-2403 (2009). doi:10.3390/ ma2042369.

3) Kazuto Tanaka, Saya Okuda, Yoshitaka Hinoue \& Tsutao Katayama, "Effect of Water Absorption on the Fiber-Matrix Interfacial Shear Strength of carbon Nanotube-Grafted Short carbon fiber Reinforced Polyamide Resin,” Journal of composite Science, 3 (1) 1-9 (2019). doi:10.3390/jcs3010004.

4) Aljaz Kovacic, Nejc Novak, Matej Vesenjak, Polona Dobnik Dubrovski, Zoran Ren, "Geometrical and mechanical properties of polyamide PA 12 bonds in Composite advanced pore morphology (APM) foam structures," Archives of Civil and Mechanical
Engineering,18(4)1198-1206(2018).

doi.org/10.1016/j.acme.2018.01.004.

5) Yan Ma, Yuqiu Yang, Toshi Sugahara, Hiroyuki Hamada, “A study on the failure behaviour and Mechanical Properties of Unidirectional fiber reinforced thermosetting \& thermoplastic composites,”

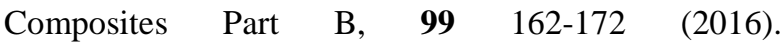
doi.org/10.1016/j.compositesb.2016.06.005.

6) Andre Duarte B.L. Ferreira, Paulo R.O. Nóvoa, Antonio Torres Marques, "Multifunctional Material Systems: A state-of-the-art review," Composite Structures, $151 \quad 3-35 \quad$ (2016). doi.org/10.1016/ j.compstruct.2016.01.028.

7) Qinghao Meng, Jinlian $\mathrm{Hu}$, “A review of shape memory polymer composites and blends," Composites: Part A, 40 (11) 1661-1672 (2009). doi.org/10.1016/ j.compositesa.2009.08.011.

8) Shang-Han Wu, Feng-Yih Wang, Chen-Chi. M. Ma, Wen-Chi Chang, Chun-Ting Kuo, Hsu-Chiang Kuan, Wei-Jen Chen, "Mechanical, thermal and morphological properties of glass fiber and short carbon fiber reinforced polyamide- 6 and polyamide6rclay nanocomposites,” Materials Letters, 49 (6) 327333 (2001). doi.org/10.1016/S0167-577X (00)00394-3.

9) Yan Ma, Masahito Ueda, Tomohiro Yokozeki, Toshi Sugahara, Yuqiu Yang, Hiroyuki Hamada, "A comparative study of the mechanical properties and failure behavior of short carbon fiber/epoxy and short carbon fiber/polyamide 6 unidirectional composites,” Composite Structures 160, 89-99 (2017). doi.org/ 10.1016/j.compstruct.2016.10.037.

10) Luca Quagliato, Yonghee Kim, Joao Henrique Fonseca, Dosuck Han, Seungjong Yun, Hyungtak Lee, Nara Park, Hyungyil Lee, Naksoo Kim, “The influence of fiber orientation and geometry-induced strain concentration on the fatigue life of short carbon fibers reinforced polyamide6," Materials and Design, 190 114 (2020). doi.org/10.1016/j.matdes.2020.108569.

11) Meltem Altin Karataş, Hasan Gokkaya, "A review on machinability of short carbon fiber reinforced polymer (SCFRP) and glass fiber reinforced polymer (GFRP) composite materials,” Defence Technology, 14 (4) 318326 (2018). doi.org/10.1016/j.dt.2018.02.001.

12) Nevin Gamze Karsli, Ayse Aytac, "Tensile and thermomechanical properties of short carbon fiber reinforced polyamide 6 composites," Journal of composite, 51 270-275 (2013). Doi: 10.1016/ j.compositesb.2013.03.023.

13) Harini Sosiati, Yankeisna Auda Shofie, Aris Widyo Nugroho, "Tensile Properties of Kenaf/E-glass Reinforced Hybrid Polypropylene (PP) Composites with Different Fiber Loading," EVERGREEN Joint Journal of Novel Carbon Resource Sciences \& Green Asia Strategy, 05 (02) 1-5 (2018). doi.org/ 10.5109/1936210.

14) Young Shin Kim, Jae Kyung Kim \& Euy Sik Jeon, "Effect of the Compounding Conditions of Polyamide 
6, Short carbon fiber, and Al2O3on the Mechanical \& Thermal Properties of the Composite Polymer," MDPI, Materials, 12(18) 01-14 (2019). doi.org/10.3390/ma12183047.

15) Honglin Luo, Guangyao Xiong, Chunying Ma, Deying Li, Yizao Wan, "Preparation and performance of long carbon fiber reinforced polyamide 6 composites injection-molded from core/shell structured pellets,” Materials \& Design 64 294-300

(2014). doi.org/10.1016/j.matdes.2014.07.054

16) Xinghui Chen, Qiang Yu, Lixia Liu, Wenhua Ji, Li Yang, and Dongli Fan, "Research on mechanical properties of short carbon fiber/ polyamide reinforced P.P. composites," AIP Conference Proceedings, $\quad 1890 \quad 1-4 \quad$ (2017). doi.org/10.1063/1.5005206.

17) A.Hassan, P.R. Hornsby, M.J. Folkes, "Structureproperty relationship of injection-molded carbonfiber-reinforced polyamide 6,6 composites: the effect of compounding routes", Polymer Testing, 22(2) 185-189 (2003). doi.org/10.1016/S01429418(02)00068-5.

18) D. Hui, R.D. Goodridge, C.A. Scotchford, B D.M. Grant, "Laser sintering of nano-hydroxyapatite coated polyamide 12 powders," Additive Manufacturing, 22 560-570 (2018). doi.org/ 10.1016/j.addma.2018.05.045

19) Chunze Yan, Liang Hao, Lin $\mathrm{Xu}$, Yusheng Shi, "Preparation, characterization, and processing of short carbon fiber/polyamide-12 composites for selective laser sintering," Composites Science and Technology,71 1834 -1841(2011).doi.org/10.1016/ j.compscitech.2011.08.013.

20) Xin Wang, Man Jiang, Zuowan Zhou, Jihua Gou, DavidHui, “3D printing of polymer matrix composites: A review and perspective,” Composites $\begin{array}{lllll}\text { Part } & \text { B, } & 110 & 442-458 & \text { (2017). }\end{array}$ doi.org/10.1016/j.compositesb.2016.11.034.

21) Parvez Alam, Colin Robert, Conchur M.O Bradaigh, "Tidal turbine blade composites - A review on the effects of hygrothermal ageing on the properties of SCFRP," Composites Part B, 149 248-259 (2018). doi.org/10.1016/j.compositesb.2018.05.003.

22) Sarower Kabir, Faiz Ahmad, Khurshid Malik, Norlin Nosbi, Laurent GUI LAUMAT, "Effect of Heat Resistant Coating on the Drilled Hole Quality of Hybrid Fiber Reinforced Epoxy Composite," EVERGREEN Joint Journal of Novel Carbon Resource Sciences \& Green Asia Strategy, 07 (04) 530-537 (2020). Doi.org/10.5109/4150472.

23) Dilip S. Choudhari, V.J.Kakhandki, "Comprehensive study and analysis of mechanical properties of chopped short carbon fiber reinforced nylon 66 composite materials," Materials Today: Proceedings, $44 \quad$ (6) 4596-4601 (2020). doi.org/10.1016/j.matpr.2020.10.828
24) A. Kausar, "Advances In Short carbon fiber Reinforced Polyamide-Based Composite Materials,” Advance in Material Science, $19 \quad$ (4) 67-82 (2019). doi:10.2478/adms-2019-0023.

25) Jinchuan Chen, Huajie $\mathrm{Xu}$, Chuntai Liu, Liwei Mi, Changyu Shen, "The effect of double grafted interface layer on the properties of short carbon fiber reinforced polyamide66,” Composites Science and Technology168 20-27 (2018). doi.org/10.1016/j.compscitech.2018.09.007.

26) Shan-Fan-Long Jin, Kyong Yop, Rhee, D avid Hui, Soo-Jin Park, "Recent advances in carbon- fiber reinforced thermoplastics composites: A review," Composites Part B, $142 \quad$ 241-250 (2018). doi:10.1016/j.compositesb.2017.12.007.

27) W. Z. Wei, J, Li \& Y.F.Zhang, “Tensile properties of surface-treated short carbon fiber reinforced ABS/PA6 composites,” Plastics, Rubber \& Composites, 39 (1) 16-21(2010). doi.org/10.1179/174328910X12608851832173.

28) M. Kalin, A.Kupec, "The dominant effect of temperature on the fatigue behavior of polymer gears," Wear, 376-377 1339-1346 (2017). doi.org/10.1016/j.wear.2017.02.003.

29) Sung Soo Kim, Min Wook Shin, Ho Jang, "Tribological properties of short glass fiber reinforced polyamide 12 sliding on medium carbon steel," Wear, 274- $275 \quad 34-\quad 42 \quad$ (2012). doi.org/10.1016/j.wear.2011.08.009.

30) Youngjae Yoo, Hoing Lae Lee, Sung Min Ha, Byung Kuk Jeon, Jong Chan Won, and Sung-Goo Lee, "Effect of graphite and short carbon fiber contents on the morphology and properties of thermally conductive composites based on polyamide 6," polymer International, 63(1) (2013). doi:10.1002/pi.4534.

31) F.P. La Mantia, M. Morreale, "Green composites: A brief review,” Composites: Part A, 42(6), 579-588 (2011). doi.org/10.1016/j.compositesa.2011.01.017.

32) Rohan Muni Bajracharya, Allan C. Manalo, Warna Karunasena, Kin-Tak Lau, “An overview of mechanical properties and durability of glass-fiber reinforced recycled mixed plastic waste composites," Materials and Design, 62 98-112 (2014). doi.org/10.1016/j.matdes.2014.04.081.

33) Seyyedvahid Mortazavian, Ali Fatemi, "Fatigue behavior and modeling of short fiber reinforced polymer composites: A literature review,” International Journal of Fatigue, 70 297-321 (2015). doi.org/10.1016/j.ijfatigue.2014.10.005.

34) Kazuto TANAKA, Shota MIZUNO, Hirokazu HONDA, Tsutao KATAYAMA \& Shinichi ENOKI, "Effect of Water Absorption on the Mechanical Properties of Short carbon fiber/Polyamide Composites”, Journal of Solid Mechanics \& Materials $\begin{array}{llll}\text { Engineering, } & 7 & \text { (5) } & \text { 520-529 }\end{array}$ doi.org/10.1299/jmmp.7.520.

35) Garima Mittal, Kyong Y. Rhee, Vesna Miskovic- 
Stankovic, David Hui, "Reinforcements in multiscale polymer composites: Processing, properties, and applications,” Composites Part B, 138122-139 (2018).doi.org/10.1016/j.compositesb.2017.11.028.

36) Muhammad Hamid Mahmood, Muhammad Sultan, Takahiko Miyazaki, "Study on Water-Vapor Adsorption onto Polymer and Carbon-Based Adsorbents for Air-Conditioning Applications," EVERGREEN Joint Journal of Novel Carbon Resource Sciences \& Green Asia Strategy, 06 (03) 32-36 (2019). doi.hdl.handle.net/2324/2349297.

37) Egiza, Mohamed, "Si and Cr Doping Effects on Growth and Mechanical Properties of Ultrananocrystalline Diamond/Amorphous Carbon Composite Films Deposited on Cemented Carbide Substrates by Coaxial Arc Plasma Deposition," EVERGREEN Joint Journal of Novel Carbon Resource Sciences \& Green Asia Strategy, 03 (01) 32-36 (2016). doi.org/10.5109/1657738.

38) Jicai Liang, Yuqiang Xu, Zhiyong Wei, Ping Song, Guangyi Chen \& Wanxi Zhang, "Mechanical Properties, crystallization and melting behavior of short carbon fiber-reinforced PA6 composites," Journal of Thermal analysis \& Calorimetry, 115 209-218 (2014). doi.org/10.1007/s10973-013-31842.

39) E.C. Botelho, Figiela, M.C. Rezendeb, B. Laukea, "Mechanical behaviour of short carbon fiber reinforced polyamide composites," Journal of composite science and technology, 63 (13) 1843 1855 (2003). doi.org/10.1016/S02663538(03)00119-2.

40) Van-Ta Do, Huu-Duc Nguyen-Tran, Doo-Man Chun, "Effect of polypropylene on the mechanical properties and water absorption of carbon-fiberreinforced-polyamide-6/polypropylene composite," Journal of composite structure, $150 \quad 240 \quad-245$ (2016). doi.org/10.1016/j.compstruct.2016.05.011.

41) Alimin Mahyudin, Syukri Arief, Hairul Abral, Emriadi, Mulda Muldarisnur, Mila Puteri Artik, “A Mechanical Properties and Biodegradability of Areca Nut Fiber-reinforced Polymer Blend Composites,” EVERGREEN Joint Journal of Novel Carbon Resource Sciences \& Green Asia Strategy, $07 \quad$ (03) 366-372 (2020). http://hdl.handle.net/2324/4068618.

42) Doo-Won Kim, Hyun-Sig Kil, Koji Nakabayashi, Seong-Ho Yoon, Jin Miyawaki, "Improvement of Electric Conductivity of Non-graphitizable Carbon Materialvia Breaking-down and Merging of the Microdomains," EVERGREEN Joint Journal of Novel Carbon Resource Sciences \& Green Asia $\begin{array}{lllll}\text { Strategy, } & 04 \quad \text { (01) } & \text { 16-20 }\end{array}$ doi.org/10.5109/1808307

43) Yi Li, Jinting $\mathrm{Xu}$, Zhiyong Wei, Yuqiang $\mathrm{Xu}$, Ping Song, Guangyi Chen, Lin Sang, Ying Chang, Jicai Liang, "Mechanical Properties and Nonisothermal
Crystallization of Polyamide 6/Short carbon fiber Composites Toughened by Maleated Elastomers," Polymer Composites, 35 (11) 2170-2179 (2014). doi.org/10.1002/pc.22881.

44) Pierre-Yves Le Gac, Bruno Fayolle, "Impact of fillers short glass fibers and rubber on the hydrolysis-induced embrittlement of polyamide66," Composites Part B, 153 256-263 (2018). doi:10.1016/j.compositesb.2018.07.028. 